\title{
Design and development of a novel acoustic rain sensor with automated telemetry
}

\author{
Maria Leonora Guico 1, *, Gemalyn Abrajano ${ }^{2}$, Prince Aldrin Domer ${ }^{3}$, and Jose Paulo \\ Talusan $^{4}$ \\ 1,2,3,4 Department of Electronics, Communications, and Computer Engineering, Ateneo de Manila \\ University, Quezon City, Philippines
}

\begin{abstract}
This paper presents the recent results of the design of a novel acoustic rainfall sensing system that is low-cost, portable, and easily deployable, which makes use of the recorded sound produced by the impact of the raindrops on the sensor surface. The sensor design allows the gathering of acoustic signal power and sending it to a server after a specified time interval, either through SMS or mobile internet connection. It exists in a weather-proof, standard-conformant, standalone system with its own power supply and telemetric capabilities. These acoustic point sensors can gather rainfall data at high spatial and temporal resolutions. Such deployments can show the variations of rainfall intensities in subkilometer areas, particularly in the tropical regions. Since it is low-cost, it can also improve the density of rainfall measuring devices in an area. Moreover, the reliability is improved by providing near-real time data, as opposed to tipping buckets with manual data retrieval. The prototype sensor system was placed next to standard rain measuring devices and observed during the rainy season. The paper will discuss the design and deployment of the system, as well as initial results of data analysis and comparison with standard rain measuring devices.
\end{abstract}

\section{Introduction}

Intensities of rain events in tropical regions vary significantly in time and space. Gathering precipitation data for these events currently makes use of rainfall estimates in place of gauge observations, even when spatial localization and sub-kilometer rain measurements are significant. Satellite imagery, for instance, captures precipitation data at 100's of kilometers which cannot provide the resolution that tipping buckets or automatic weather stations can at sub-kilometer regions. Further, rain gauges in tropical countries are critical for facilitating the provision of an alarm or warning system for areas where phenomena such as flooding or landslide frequently occur. Placing these sensors at critical points detect if rain rates in high-risk areas - urban and rural, alike - are exceeding threshold levels. However, in a developing

\footnotetext{
Corresponding author: mguico@ateneo.edu
} 
country such as the Philippines, more economical measures for risk-mitigation in raininduced calamities must be employed

A novel means of monitoring rain events was developed by the Department of Electronics, Computer and Communications Engineering of the Ateneo de Manila University. Through the use of standalone acoustic point sensor, shown in Figure 1(a), for rainfall data gathering,

the sound produced by the impact of the raindrops on the sensor surface captured by the microphone input can provide information on rain events, where levels of generated sound

corresponds to rain intensities. The research and development of this type of rain sensor started a few years back, and has not been performed by other research groups [1-2]. Similar efforts do exist but makes use of piezoelectric detectors to capture acoustic data [3]. Further, no data gathering from field deployments in actual weather observation sites has been done on the acoustic rain sensor. It has since undergone hardware and software design changes, from initial research prototypes [4], to improve reliability and operation to survive harsh weather and environmental field conditions.

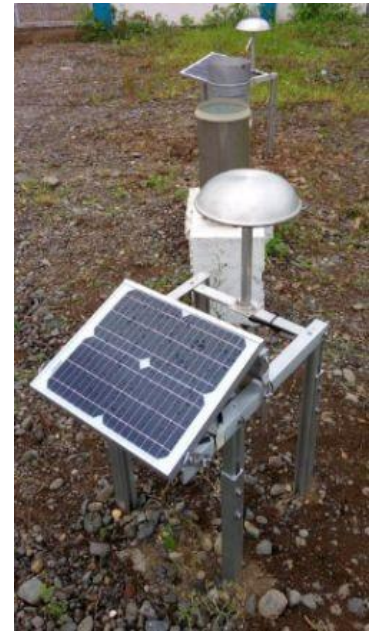

(a)

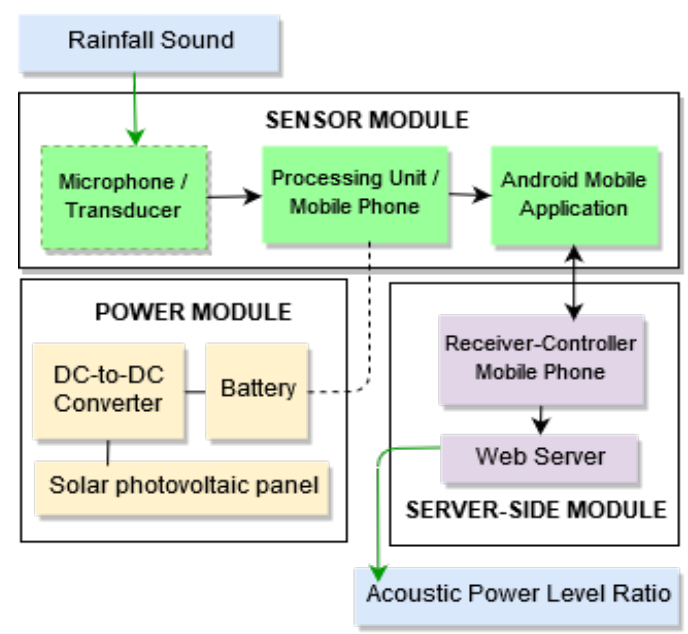

(b)

Fig 1. (a) Acoustic rain sensor hardware setup, and (b) overview of its operation

\section{Materials and Methods}

\subsection{Materials}

The acoustic rain sensor presents a practical solution to rain monitoring that addresses problems related to cost-effectiveness, portability, and scalability. It works by recording the sound of the rainfall as it hits the metal sensor casing which doubles as an enclosure to the microphone input connected to the mobile phone (see Figure 1(b) for the operation overview). The metal enclosure is attached to a main frame which acts as a mounting platform and is composed of four legs made of aluminum metal bars for a sturdy and corrosion-resistant emplacement. A 12-Watt solar photovoltaic panel is placed atop the main frame at a 45-degree angle for maximized sun exposure. This doubles as 
waterproofing of the casing, reinforced by a silicone sealant, that houses the mobile phone and power supply elements (a 5-A DC-to-DC converter, and a 20000-mAh lithium-ion battery), which are responsible for the power supply, data processing, and data telemetry functions of the whole sensor setup.

The mobile phone in the acoustic rain sensor is an Android-based device which allows it to leverage high processing capacity using the Android software development kit (SDK). A 1.2-MB mobile application, shown in Figure 2, was developed to customize functions the phones can perform, both for the receiver and transmitter end, where managing the audio resource for the application to record audio from the microphone is handled by the AudioRecord public class. Deployed mobile phones in the field are labeled as 'transmitters' while a control phone in the laboratory functions as the 'receiver'. The transmitter and controller-receiver mobile phones are all identified by the numbers in the installed subscriber identity module (SIM) cards; all transmitter devices can be remotely controlled by the controller-receiver device through specific Short Message Service (SMS) messages. A list of instructions that the transmitter devices can recognize are listed below in Table 1.

Table 1. Control SMS messages from controller-receiver to transmitter devices

\begin{tabular}{|c|c|}
\hline 'Start-SMS' & $\begin{array}{c}\text { Initiates operation of the transmitter device: } \\
\text { recording of sound, audio processing and telemetry. }\end{array}$ \\
\hline 'Stop-SMS' & Stops the operation of the transmitter device. \\
\hline $\begin{array}{c}\text { 'promo- }<\text { Message }>- \\
<\text { Number }>\text { ' }\end{array}$ & $\begin{array}{c}\text { Allows SMS messages to be sent by the transmitter } \\
\text { device to the specified number; used for registering to } \\
\text { network provider's value-added service. }\end{array}$ \\
\hline
\end{tabular}
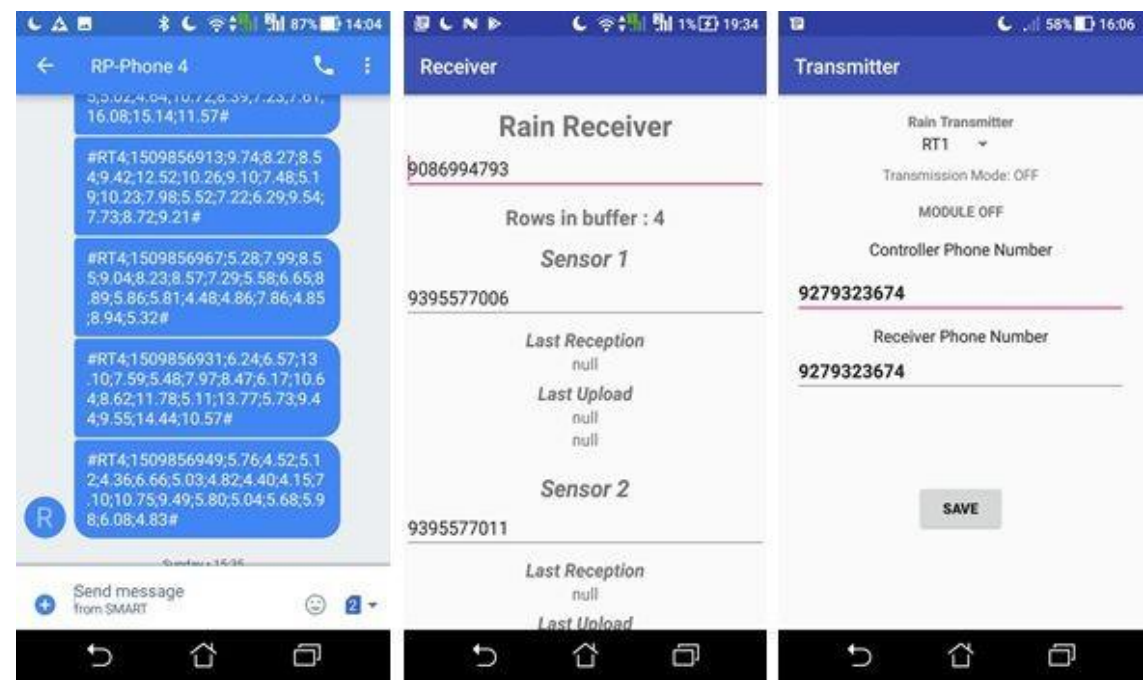

Fig 2. (left to right) Screenshots of (1) an acoustic rain sensor data sent as an SMS, the Android application in the (2) controller-receiver, and (3) transmitter end. 


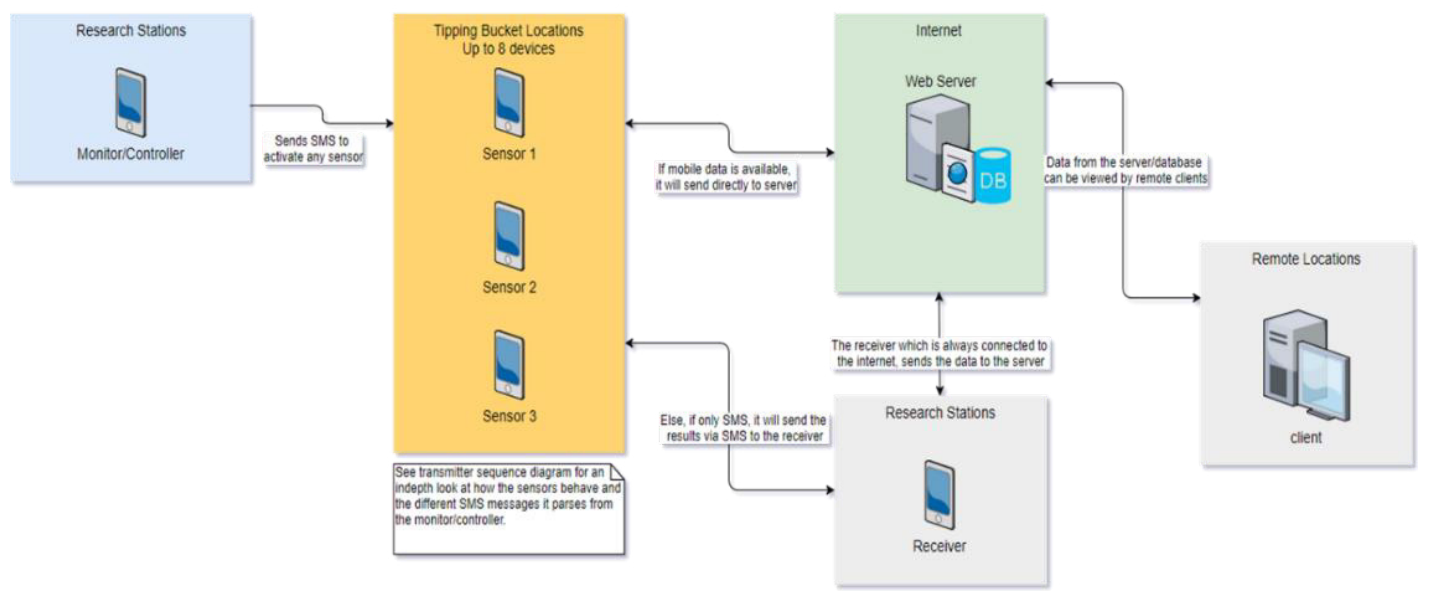

Fig 3. Outline of the telemetry system from the transmitter device in the sensor setup to the web server or the receiver phone.

\subsection{Methods}

The specific system used in this acoustic rain sensor is outlined in Figure 3. An SMS message is first sent to the transmitters to activate operation. From there, the transmitter will start accepting and processing audio input as triggered by a rain event. The sound is recorded as a 16-bit audio sampled at $44,100 \mathrm{~Hz}$. The corresponding acoustic power level is then calculated as the variance of the samples, and is returned as a power ratio value in decibel $(\mathrm{dB})$, available for every second. This value is sent directly to a web server and is stored in its designated transmitter cache, if mobile internet service is available. Otherwise, the data will be sent via SMS to a receiver phone that is always connected to the Internet, which will then be uploaded to the web server. Data sent through SMS is no longer queued for telemetry, once mobile internet service becomes available again.

Sending of data from the transmitter devices are enabled by SMS messaging and mobile internet services, which are availed to the transmitter devices by a special instruction (see Table 1). All the data stored in the server can then be accessed and viewed by remote clients, and can be downloaded as a comma separated value (.CSV) file. In one deployed sensor setup, the 16-bit pulse code modulation (.PCM) files are also saved to the mobile phone's internal memory, containing 5 minutes of raw audio recording for each file. The PCM files are to be used for digital signal processing to identify and investigate signal parameters.

Table 2. Deployment sites and their profile

\begin{tabular}{|c|c|}
\hline Diliman, Quezon City & $\begin{array}{c}\text { Situated near a residential area; possible source } \\
\text { of ambient noise due to human activity. }\end{array}$ \\
\hline Infanta, Quezon City & $\begin{array}{c}\text { Situated near a rice farm; no nearby source } \\
\text { of loud ambient noise. }\end{array}$ \\
\hline
\end{tabular}




\begin{tabular}{|c|c|}
\hline Legazpi City, Albay & $\begin{array}{c}\text { Situated near a major service road, and a local } \\
\text { airport; with possible source of loud ambient noise. }\end{array}$ \\
\hline Sasa, Davao del Sur & $\begin{array}{r}\text { Situated near a local airport ; with possible } \\
\text { source of loud ambient noise }\end{array}$ \\
\hline
\end{tabular}

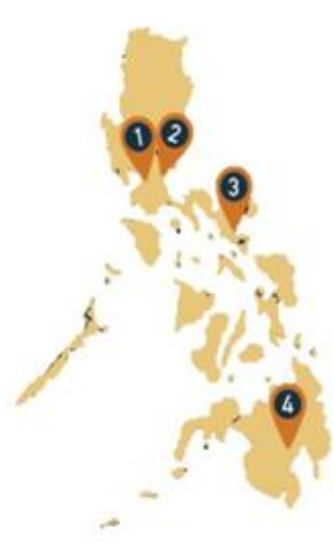

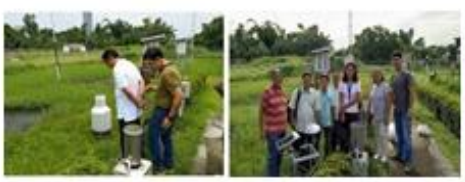

Site 1: Diliman, Quezon City

(June 27, 2017)
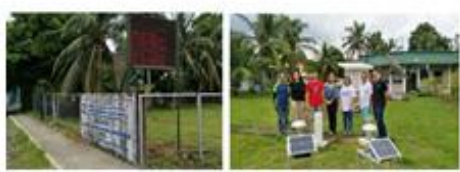

Site 3: Legazpi City, Albay

(September 22, 2017)
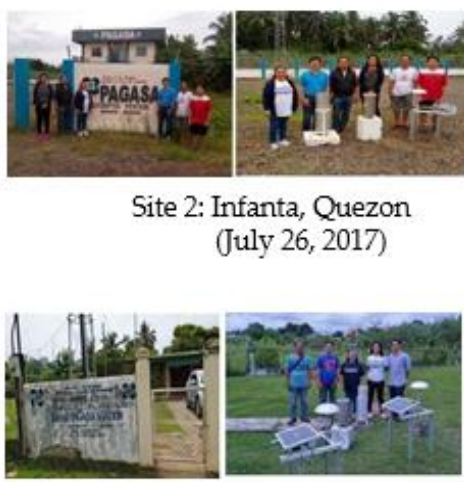

Site 4: Sasa, Davao del Sur (August 18, 2017)

Fig 4. Location of Philippine Atmospheric and Geophysical Services Administration (PAGASA) weather observation sites where the acoustic rain sensors are deployed.

The rain sensors are deployed in four weather observation (Table 2, Figure 4) sites in the Philippines starting June 2017. For purposes of comparing with existing conventional rain gauges, the rain sensors are also placed side by side with tipping buckets with the main sensing element leveled with the brim of the rain gauge. Data retrieval from the tipping bucket data logger was likewise introduced in the data gathering setup using a Raspberry Pi with a real-time clock (RTC) that sends recorded tipping event to a server via Internet over a WiFi network. Recorded tipping events are to be used for mapping acoustic rain sensor output to tipping bucket rain gauge data.

\section{Results and Discussion}

The performance of the data gathering setup as deployed in the field are evaluated, which includes both the acoustic rain sensor and the tipping bucket data logger. Checking of gathered acoustic rain sensor data, and validation of rain events through the records in the tipping buckets data loggers allowed the evaluation of performance of the sensor telemetry system; whereas the validation of tipping bucket logger data was done in reference to weather observation data. Further, initial mapping of sensor output with the retrieved tipping bucket rain gauge output shows positive correspondence between rain intensities and computed acoustic power levels, especially for moderate to heavy rains. 


\subsection{Field Deployment Results}

The aluminum frame and sensor casing deployed in the field experienced no rain-induced corrosion. No water leaks inside the circuit box and the microphone casing occured. Protection from foreign elements was likewise achieved proving the reliability of the sensor body design, and observed protocols in sealing sensitive sensor elements.

Additionally, the configuration of the power supply allowed for continuous charging of the battery in the setup thereby allowing continuous operation of the mobile phones. Except for one sensor setup where the Pulse Code Modulation (.PCM) files of the recorded sound are saved in the internal memory of the mobile phone; overloading and eventual crashing of the device is encountered, in which case, the PCM files were manually retrieved, and the Android application was re-activated. This sensor setup is located near the research laboratory to accommodate logistical constraints. Further, the status of phones - whether on or off - are validated through checking data transmitted in the server and by making a call to the transmitter device from the receiver.

Meanwhile, data reception is evaluated to be affected by the poor network performance of the selected cellular network service provider. Preparation of data summaries showed that acoustic rain sensor data for some rain events were not successfully or consistently sent to the web server. Additionally, occasional delays in data reception were encountered, in which case, chronological ordering of the received data were performed.

Lastly, the Raspberry Pi modules attached to the tipping bucket data logger was operational for the entire data collection period. Time-stamped tipping events were recorded and uploaded as a comma separated value (.CSV) file to a file hosting service. Sent files were grouped by day and were uploaded by the end of the day, including dates with no tipping events.

\subsection{Acoustic Rain Sensor Output Versus Tipping Bucket Rain Gauge}

Gathered acoustic rain sensor data from latest deployments returned values from $1-56 \mathrm{~dB}$. To check trends or correspondence of returned values with the tipping bucket rain gauge, recorded tipping events were converted into rain rates using the novel International Atmospheric Physics (IAP) technique [5]. Rain rate extraction using the novel IAP technique, shown in equation (1) below, demonstrates that the time difference for two consecutive tipping events is taken into account, and where the sum of tips is defined for the time duration being considered. A unit tip is taken to be equivalent to $0.5 \mathrm{~mm}$, as specified by the tipping bucket rain gauge collocated with the acoustic rain sensors. In general, there is a positive correspondence between the extracted rain rates and the gathered acoustic power levels for medium to heavy rain events.

$$
\text { Rain Rate }=\frac{\Delta \text { sum of tips }}{\Delta \text { time }}\left(\frac{3600 * 0.5 \mathrm{~mm}}{\mathrm{hr} .}\right)
$$

Figure 5 shows sample visualizations of gathered data for a 5-hour rain event in Infanta, Quezon weather observation site last November 24, 2017. The moving average with a window size of 60 is shown as the red trendline of the received acoustic power level ratio $(\mathrm{dB})$, while the horizontal lines in the rain rate graph represent thresholds of different rain intensity levels specified by PAGASA (see Table 3). It can be observed 
that for periods of very intense rainfall, the acoustic rain sensor returns high $\mathrm{dB}$ values, with minimum dispersion. That is, the values are clustered at certain levels; whereas for lower intensities of rainfall, the $\mathrm{dB}$ values are generally more scattered, subject to the characteristic of the ambient noise in the environment, on top of intermittent man-made or biological noises present. In Figure 5, this is demonstrated after the 22:00 mark where the rain rate graph signifies very low-intensity rainfall where the $\mathrm{dB}$ values are evidently more dispersed. In general, it can be observed that the acoustic rain sensor plot prominently traces the trend of the computed rain rates.

It follows that when there is presence of strong rainfall, the impact of the rainfall drops on the sensor casing dominates the sound field that the microphone captures. This means that the ambient noise, during periods of strong rainfall is registered less. In the same logic, during periods of low intensity rainfall, the sound field is distributed across different sources of noise in the surrounding environment. Therefore, the sound captured by the microphone during these times is reliant on the sound profile of the selected deployment site (see Table 2).

Table 3. PAGASA Rain Rate Classification and Color

Representation of Threshold Lines in the Rain Rate Plot

\begin{tabular}{|c|c|c|}
\hline Rain Type & $\begin{array}{c}\text { Intensity } \\
\mathbf{( m m} / \mathbf{h r} \mathbf{)}\end{array}$ & Threshold (Color) \\
\hline Torrential & $>30$ & Red \\
\hline Intense & $15-30$ & Orange \\
\hline Heavy & $7.5-15$ & Green \\
\hline Moderate & $2.5-7.5$ & Blue \\
\hline Light & $0.01-2.5$ & - \\
\hline
\end{tabular}
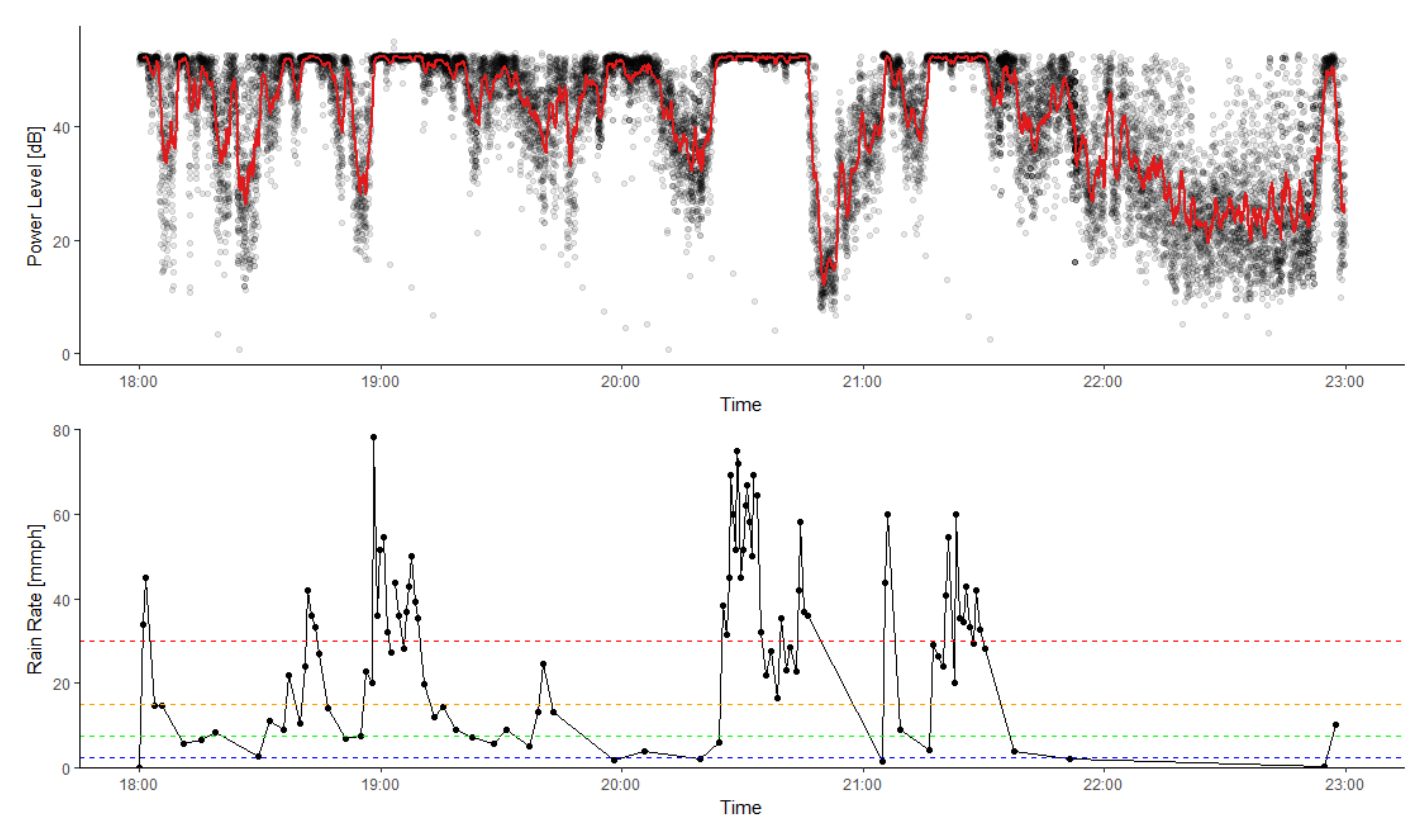
Figure 5. Sample rain rate and acoustic power level visualization for a 5 -hour rain event in Infanta, Quezon weather observation site last November 24, 2017

\section{Conclusion}

The design of the acoustic rain sensor prototype is motivated by the need to lower the materials cost, without compromising reliability amidst harsh weather and environmental conditions the sensors are expected to be subjected to. Further, the decision to go with the implemented telemetry system stems from the need to come up with a way to integrate more readily available network service in the selected field deployment sites where Internet service through hardwired and wireless broadband access is limited or not present at all. Sending data through SMS messages proved to be an easy but not very dependable way to retrieve gathered data, owing to its reliance on the quality of service of cellular network service providers, alongside the degradation of service due to signal attenuation in the presence of localized heavy rain events. Further, while telemetry through mobile internet service has better performance than through SMS, it is still subject to the availability of the service, which can be quite unreliable at times.

Even so, successfully retrieved data showed positive correspondence with computed rain rate values. The acoustic rain sensor is capable of tracing the trend of rain rates computed from the tipping bucket rain gauges and is more prominent in rain events with higher intensity and in less noisy environments. In particular, periods of very intense rainfall correspond to high values of gathered power level ratio further characterized by minimum dispersion. With low-intensity rain events, the rain sensor picks up ambient noise more, as characterized by the profile of the environment generating different sound sources the microphone input can capture. In general, the gathered power level ratios are scattered across the whole range of available $\mathrm{dB}$ values. Findings in this research presents additional considerations that must be taken into account in the ongoing data analysis, and possible future deployments.

This research is a one-year partnership project between Ateneo de Manila University (ADMU) and the Philippine Atmospheric and Geophysical Services Administration (PAGASA) funded by the Department of Science and Technology (DOST) - Philippine Council for Industry, Energy and Emerging Technology Research and Development (PCIEERD) and partially funded by the Ateneo de Manila University. Acknowledgment also goes to Ms. Catherine Dela Cruz (ADMU), Engr. Juan Antonio Marinas, M.S. (ADMU), Mr. Chrisandro Favila (ADMU), Mr. Sonny Toledo (ADMU), Engr. Jeric Lim (ADMU), Ms. Danilyn Joy Orioste (ADMU), Mr. Francis Jerome Tiausas (ADMU), Engr. Ferdinand Barcenas (PAGASA), and Engr. Wilfredo Tuazon (PAGASA) for the rendered support in this research project.

\section{References}

1. G. Gu, R. Adler, G. Huffman and S. Curtis, Long-Term Increase in Rainfall Seen in Tropics, v. 20, no.15, Journal of Climate, (2007).

2. N. J. C. Libatique, G. L. Tangonan, R. Gustilo, et.al Design of a Tropical Rain-Disaster Alarm System: A New Approach based on Wireless Sensor Networks and Acoustic Rain Rate Measurements. International Instrumentation and Measurement Technology Conference 2009 (I2MTC), Singapore, (2009). 
3. N. de Giesen, C. Degen, and R. Hut. Affordable acoustic disdrometer: Design, calibration, tests.Delft University of Technology. American Geophysical Union, Fall Meeting, (2009).

4. E. M. Trono, M. L. Guico, et.al. Rainfall monitoring using acoustic sensors. TENCON 2012 IEEE Region 10 Conference, Cebu, 2012, pp. 1-6, (2012)

5. O. Fiser and O. Wilfert. Novel processing of tipping bucket rain gauge records. Atmospheric Research, Volume 92, Issue 3, (2009) 